CLINICAL STUDY

\title{
The role of vitamin $D$ in metabolic disturbances in polycystic ovary syndrome: a systematic review
}

\author{
Y H M Krul-Poel ${ }^{1}$, C Snackey ${ }^{1}$, Y Louwers ${ }^{2}$, P Lips ${ }^{3}$, C B Lambalk ${ }^{4}$, J S E Laven ${ }^{2}$ and S Simsek ${ }^{1,3}$ \\ ${ }^{1}$ Department of Internal Medicine, Medical Centre Alkmaar, PO Box 501, 1800 AM Alkmaar, The Netherlands, ${ }^{2}$ Division of Reproductive \\ Medicine, Department of Obstetrics and Gynaecology, Erasmus Medical Centre, PO Box 2040, 3000 CA Rotterdam, The Netherlands, \\ Departments of ${ }^{3}$ Internal Medicine/Endocrinology and ${ }^{4}$ Obstetrics, Gynaecology and Reproductive Medicine, VU University Medical Centre, \\ PO Box 7057, 1007 MB Amsterdam, The Netherlands \\ (Correspondence should be addressed to S Simsek at Department of Internal Medicine, Medical Centre Alkmaar; Email: s.simsek@mca.nl)
}

\begin{abstract}
Context: Metabolic disturbances, in particular, insulin resistance (IR) and dyslipidemia, are common in women suffering from polycystic ovary syndrome (PCOS). Evidence is accumulating that vitamin D status may contribute to the development of metabolic disturbances in PCOS.

Objective: The aim of this study was to carry out a systematic review addressing the association between vitamin D status, vitamin D receptor polymorphisms, and/or polymorphisms related to vitamin D metabolism and metabolic disturbances in women with PCOS.

Design and methods: A systematic search of electronic databases was carried out up to January 2013 for observational studies and clinical trials in women suffering from PCOS with outcome measures that were related to vitamin D status. We conducted univariate and multivariate regression analyses of the weighted means to gain insights into the association between vitamin D, BMI, and IR based on existing literature.

Results: We found 29 eligible trials with inconsistency in their results. One well-designed randomized controlled trial has been carried out until now. Univariate regression analyses of the weighted means revealed vitamin D to be a significant and independent predictor of IR in both PCOS and control women. The significance disappeared after adjustment for BMI in PCOS women.

Conclusions: Current evidence suggests an inverse association between vitamin D status and metabolic disturbances in PCOS. Owing to the heterogeneity of the studies, it is hard to draw a definite conclusion. The causal relationship between vitamin D status and metabolic disturbances in PCOS remains to be determined in well-designed placebo-controlled randomized clinical trials.
\end{abstract}

European Journal of Endocrinology 169 853-865

\section{Introduction}

Polycystic ovary syndrome (PCOS) is the most common endocrine disorder in women of reproductive age, with a prevalence of $6-10 \%$ in the general population. PCOS is characterized by the following: i) ovulatory dysfunction resulting in oligo- and/or anovulation, ii) hyperandrogenism and/or hirsutism, and iii) the presence of polycystic ovarian morphology (1). PCOS is by far the most common cause of anovulatory infertility and has been reported to be associated with insulin resistance (IR), hyperinsulinemia, dyslipidemia, and central obesity $(2,3,4)$, which are all risk factors for the metabolic syndrome, type 2 diabetes mellitus, and cardiovascular disease. Metabolic disturbances are common in women suffering from PCOS: 30-40\% have impaired glucose tolerance and IR with compensatory hyperinsulinemia, and as many as $10 \%$ will have type 2 diabetes mellitus by their fourth decade $(5,6,7)$. A recent meta-analysis has revealed that dyslipidemia occurs more frequently in PCOS women than in control women: women with PCOS had higher LDL-cholesterol and non-HDL-cholesterol levels, regardless of BMI (4).

Current evidence suggests that IR has a central role in the pathogenesis of PCOS, contributing to both metabolic and reproductive disturbances (3).

Many studies have been conducted to clarify the mechanism of metabolic disturbances, in particular IR, in women affected by PCOS. In part, IR might be due to obesity. However, a substantial number of lean women affected by PCOS have IR as well, independent of obesity $(8,9)$. Recently, vitamin D deficiency has been proposed as the possible missing link between IR and PCOS. This assumption is supported by the finding that the active vitamin D-vitamin D receptor (VDR) complex regulates over 300 genes, including genes that are important for glucose and lipid metabolism as well as blood pressure regulation (10). Moreover, there is an association between poor vitamin D status and IR in patients with type 2 diabetes mellitus $(11,12,13,14,15)$. Still, it 
remains unclear whether vitamin D and IR are causally interrelated or whether they constitute two independent characteristics in women with PCOS. A recently published review carried out by Thomson et al. (16) about the role of vitamin D in the etiology and management of PCOS suggests that there is an association between vitamin D status and hormonal and metabolic dysfunctions in PCOS. However, literature about the association between vitamin D status and metabolic and hormonal disorders in women suffering from PCOS is scarce, and it has provided the scientific community with conflicting results. Therefore, we carried out this systematic review to examine the following: i) the association between vitamin $\mathrm{D}$ status and metabolic disturbances and/or endocrine parameters in women with PCOS; ii) the effect of vitamin D supplementation on metabolic disturbances and/or endocrine parameters in PCOS; and iii) the influence of VDR polymorphisms in women with PCOS.

\section{Subjects and methods}

\section{Search strategy}

Two authors independently carried out a formal computer-assisted search for observational studies and clinical trials that investigated the association between vitamin D status or VDR polymorphisms and endocrine or metabolic parameters in PCOS women. This search was confined to the MEDLINE database, Embase, and The Cochrane Library and included English-language literature up to January 2013. The following search terms were used: polycystic ovary syndrome, polycystic ovaries, vitamin D, cholecalciferol, 25-hydroxyvitamin D (250HD), vitamin D deficiency, vitamin D receptor, vitamin D receptor polymorphisms, and related terms. The detailed search strategy is given in Supplementary information, see section on supplementary data given at the end of this article.

\section{Outcome of interest and criteria for inclusion}

The primary objective of this systematic review was to examine the role of vitamin $\mathrm{D}$ in metabolic disturbances in women affected by PCOS. Studies that met the following criteria were included in this systematic review: i) inclusion of women with PCOS, with or without a control group and ii) inclusion of vitamin D status or VDR polymorphisms or polymorphisms related to vitamin $\mathrm{D}$ metabolism and their correlation with metabolic and/or endocrine parameters as the outcome of interest. We excluded letters, abstracts, and conference proceedings that were not published in fully peer-reviewed journals. For study inclusion, PCOS was defined by the presence of a combination of oligo- or anovulation, PCO morphology, and hyperandrogenism, according to the National Institutes of Health (NIH) criteria or the Rotterdam criteria (17).

\section{Data extraction}

The following data were extracted (in case they were available): author name, study design, publication year, study location, sample size, criteria used for the diagnosis of PCOS, inclusion and exclusion criteria for cases and controls, characteristics of the study population, anthropometric parameters, metabolic parameters, endocrine parameters, serum 25-hydroxyvitamin D (250HD), and method used for the measurement of serum 250HD levels and IR (defined as homeostasis model assessment-IR (HOMA-IR)). To avoid multiple-publication bias, we excluded publications with overlapping patient populations in the regression models.

\section{Statistical analysis}

Statistical analyses were carried out using the SPSS Software (version 20.0, SPSS, Inc.). Mean values of serum 250HD, HOMA-IR, and BMI were employed if available in the reviewed articles. These data were weighted by the number of participants included in the study. In case the mean values were not reported, the median was used if available. Linear regression models of the weighted means were used to assess the independent relationships between serum vitamin D, BMI, and IR in women affected by PCOS and controls. First, univariate analyses and subsequently multivariate analyses with HOMA-IR as an independent variable and serum 250HD and BMI as dependent variables were carried out. A $P$ value $<0.05$ was considered statistically significant.

\section{Results}

\section{Identified studies}

The initial literature search yielded 114 hits, 25 of which were duplicates, resulting in 89 non-duplicated publications. Of these, 52 studies were excluded based on the title or abstract and eight studies were excluded based on the lack of inclusion criteria (Fig. 1). In total, 29 studies were included in this systematic review.

\section{The association between vitamin D status and metabolic disturbances in PCOS in observational studies}

Twelve studies had an observational design $(18,19,20$, $21,22,23,24,25,26,27,28,29)$, employing a crosssectional or case-control design, with vitamin D status as one of the preliminary outcomes. A summary of these studies is given in Table 1 . The studies included premenopausal women (14-50 years) of various ethnicities, with the number of participants ranging from 45 to 400 . Different stratifications were used: seven studies 


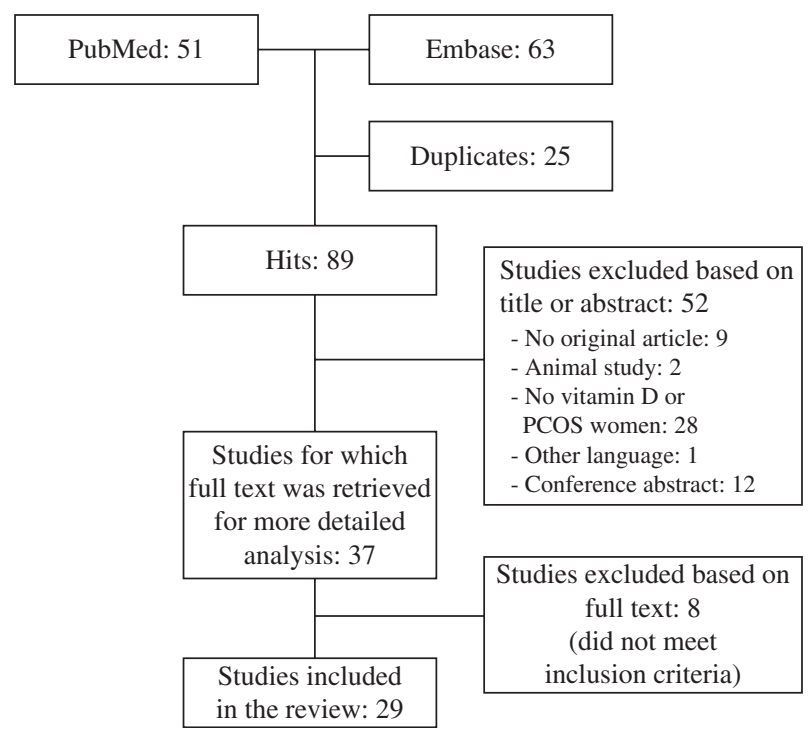

Figure 1 Flow chart for systematic review.

compared PCOS women with controls (19, 20, 22, 23, $24,27,28)$, three studies compared lean vs obese PCOS women $(18,21,26)$, one study compared PCOS women with and without the metabolic syndrome (25), and one study solely included PCOS women (29). Large differences were found in the prevalence of vitamin $\mathrm{D}$ deficiency (defined as serum $250 \mathrm{HD}$ levels $<50 \mathrm{nmol} / \mathrm{l}$ ), varying from $37 \%$ in a study carried out in Italy (21) to $72 \%$ in a study carried out in Scotland (19).

The studies that compared serum 250HD levels between PCOS and control women yielded conflicting results. In detail, three studies demonstrated a significantly lower serum 250HD level in PCOS women: 32.4 vs $73.7 \mathrm{nmol} / \mathrm{l}$ in $90 \mathrm{PCOS}$ women and 47 control women (24), 30.0 vs $43.7 \mathrm{nmol} / \mathrm{l}$ among 103 PCOS women and their controls (28), and 17.7 vs $79.2 \mathrm{nmol} / \mathrm{l}$ in $30 \mathrm{PCOS}$ women and 15 control women (27). By contrast, another study among 291 PCOS women and 109 control women demonstrated a lower serum 250HD level in control women than in PCOS women (53.5 vs $73.7 \mathrm{nmol} / \mathrm{l})(23)$.

Eleven of all the included observational studies investigated the correlation between vitamin D status and IR $(18,19,21,22,23,24,25,26,27,28,29)$. Most of these studies used HOMA-IR as an indicator of IR. Five studies reported insulin sensitivity using the quantitative insulin-sensitivity check index $(18,19,22$, 25, 27). In one study carried out by Muscogiuri et al. in 23 obese and 15 lean PCOS women, IR was evaluated using the hyperinsulinemic-euglycemic clamp (HEC) method, the gold standard for the determination of IR. The authors found a positive correlation between serum 250HD levels and glucose uptake during HEC $(r=0.33 ; \quad P=0.03)$. Serum 250HD levels were inversely correlated with BMI $(r=-0.49 ; P=0.04)$, waist circumference $(r=-0.41 ; P=0.008)$, and total fat mass $(r=-0.47 ; P=0.02)$. In a multivariate analysis, the authors reported that only total fat mass was an independent predictor of serum 250HD (21).

Nine studies found an inverse correlation between serum 25OHD levels and IR and/or insulin sensitivity in PCOS women $(18,19,22,23,24,25,26,27,29)$. However, in one of these studies, this correlation was BMI dependent (23), and four studies did not adjust for BMI or obesity $(18,22,24,26)$. Two studies carried out a multivariate analysis including serum $250 \mathrm{HD}$ as an explanatory variable regarding $\operatorname{IR}(22,25)$. Both the studies demonstrated serum $250 \mathrm{HD}$ to be an independent predictor of IR $(P=0.007, P=0.036)$. In the latter study, 206 PCOS women were stratified by hypovitaminosis $\mathrm{D}(<75 \mathrm{nmol} / \mathrm{l}, n=150)$ and vitamin $\mathrm{D}$ sufficiency ( $\geq 75 \mathrm{nmol} / \mathrm{l}, n=56$ ). The authors reported that the hypovitaminosis D group had a significantly higher HOMA-IR (1.96 vs $1.11, P=0.002$ ) than the vitamin D-sufficient group. However, after a subsequent analysis in lean and obese PCOS women stratified by hypovitaminosis $\mathrm{D}$ and sufficient vitamin $\mathrm{D}$, no significant difference was found in HOMA-IR (25).

The studies that compared serum 250HD levels between obese and lean women suffering from PCOS observed a significantly lower serum $25 \mathrm{OHD}$ level in obese PCOS women $(18,21,23,24,26)$.

\section{The effect of vitamin D supplementation in women with PCOS}

Ten intervention trials had been carried out and published until our search in January 2013 (30, 31, $32,33,34,35,36,37,38,39,40)$. Among these, four had a randomized controlled trial (RCT) design (30, 35, $36,40)$, five an open-labeled single-arm design $(32,33$, $37,38,39)$, and one a case-control study design (31). One RCT carried out by Ardabili et al. had a randomized placebo-controlled double-blind study design in which the effect of vitamin D3 supplementation on metabolic parameters and cardiovascular risk factors was evaluated in 50 PCOS women. This trial resulted in two articles included in this review $(30,34)$. In their RCT, Sathyapalan et al. (36) examined the effect of atorvastatin vs placebo on serum vitamin $\mathrm{D}$ and subsequently studied the effect of an increase in serum vitamin D levels on metabolic and endocrine parameters in PCOS women. The third RCT carried out by Rashidi et al. (35) was a pilot study in which 60 infertile PCOS women were randomized into groups treated with: i) calcium and vitamin D3; ii) calcium, vitamin D3, and metformin; or iii) metformin. They assessed effects on folliculogenesis and menstrual cycle. Bonakdaran et al. (40) carried out a RCT among 51 women in which active vitamin D (calcitriol) was administered and compared with metformin treatment and placebo.

The number of participants included in the intervention trials ranged from 11 to 100 , and all were premenopausal PCOS women. Different treatment protocols 


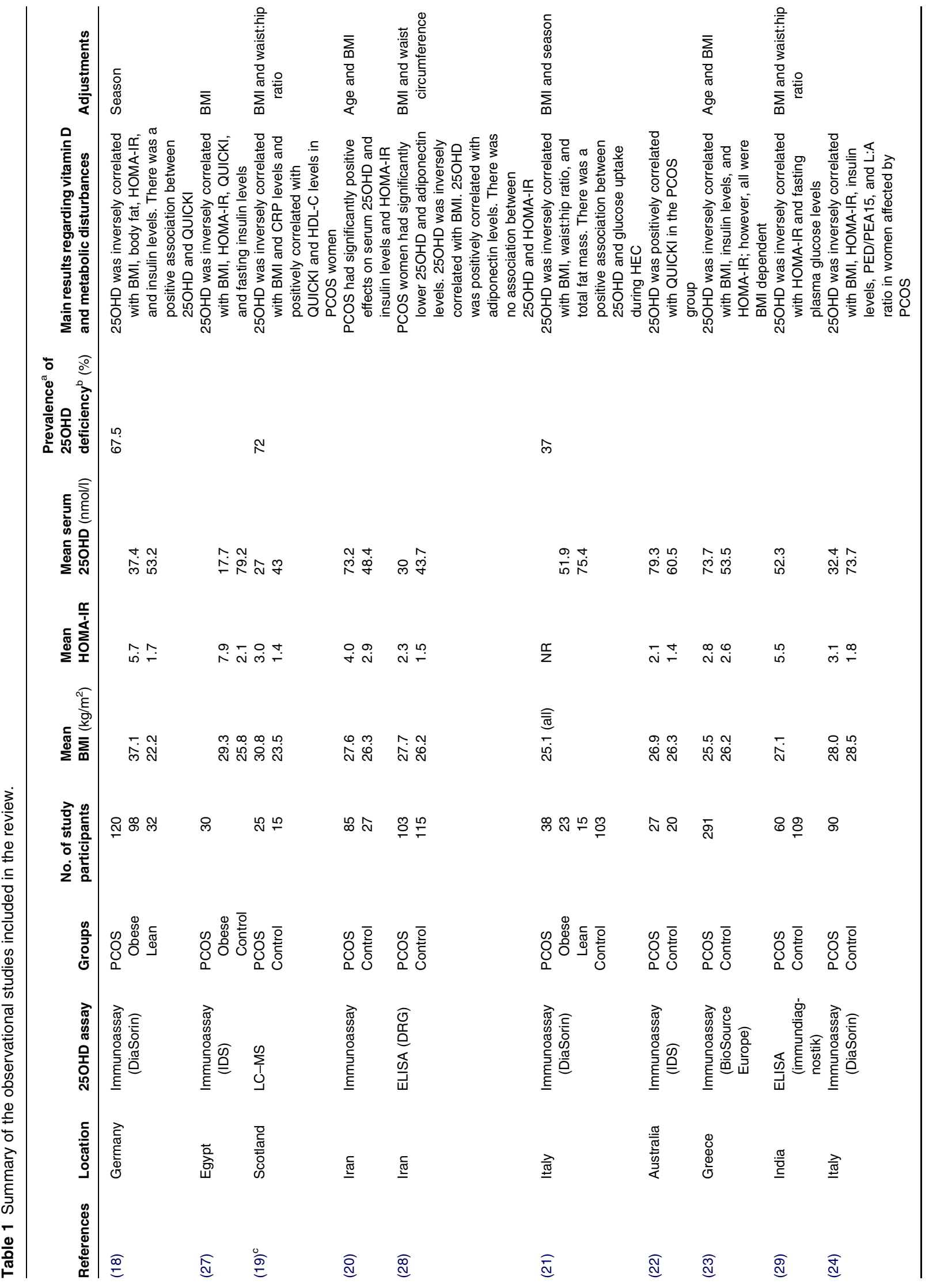




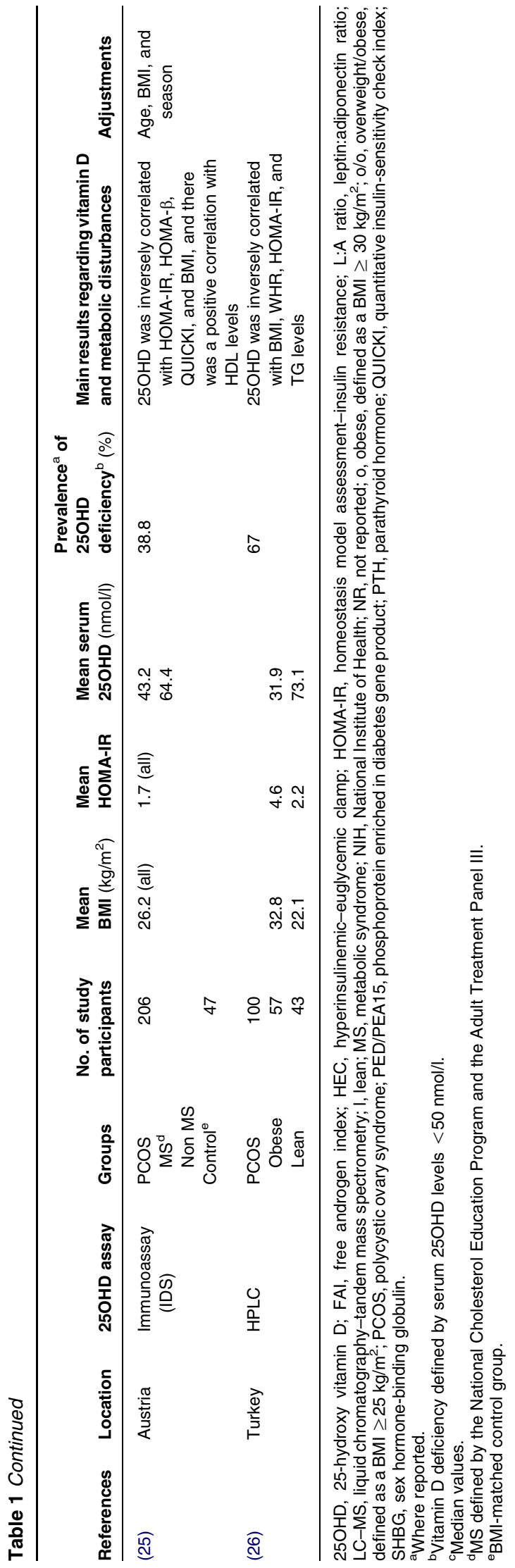

of vitamin D supplementation were used in the trials varying from 400 IU a day in the trial carried out by Rashidi et al. (35) to a single oral dose of $300000 \mathrm{IU}$ cholecalciferol in that carried out by Selimoglu et al. (37). The follow-up duration ranged from 3 weeks to 6 months. A summary of the studies is given in Table 2. In detail, the first study carried out in 1999 by Thys-Jacobs et al. (38) among 13 PCOS women, who were treated with $50000 \mathrm{IU}$ ergocalciferol weekly or biweekly to achieve a target serum 250HD level of 75-100 nmol/l, demonstrated an improvement in menstrual regularity and acne, and two women became pregnant in a follow-up period of 6 months. In addition, a more recent study carried out in Iran including 60 infertile PCOS women has found an improvement in menstrual regularity after 3 months of supplementation with $1000 \mathrm{mg} /$ day calcium and 400 IU/day vitamin D (35).

Seven intervention trials examined the effect of vitamin D supplementation on IR and other metabolic characteristics $(30,32,33,36,37,39,40)$. The only randomized placebo-controlled trial that administered vitamin D3 and examined metabolic disturbances as primary prespecified outcomes did not indicate a significant effect on IR after the administration of three oral doses of vitamin D3 in 2 months. The increase in serum $250 \mathrm{HD}$ levels was $40 \mathrm{nmol} / \mathrm{l}$ in the vitamin D group (30). The authors did find a significant increase in insulin secretion in the vitamin D group, but compared with the placebo group, this was not significant. Total cholesterol, triglyceride, and very LDL (VLDL) levels were decreased in the intervention group after vitamin D supplementation, but no effect on BMI and LDL and HDL levels was observed. The RCT carried out by Bonakdaran et al. (40), in which calcitriol was administered, also failed to find an effect on IR. In the calcitriol group, serum $250 \mathrm{HD}$ levels increased from 29 to $51 \mathrm{nmol} / \mathrm{l}$. HOMA-IR decreased from 4.2 to 2.7 ; however, this was not significant.

The other intervention trials yielded conflicting results: Selimoglu et al. (37) observed a significant decrease in HOMA-IR after the administration of a single oral dose of $300000 \mathrm{IU}$ cholecalciferol, whereas the others failed to find a positive effect on IR after the use of different regimens of vitamin D replacement $(32,33,36,39)$.

\section{The influence of VDR polymorphisms and polymorphisms related to vitamin $D$ metabolism in women with PCOS}

Literature assessing VDR polymorphisms and/or polymorphisms related to vitamin D metabolism in women suffering from PCOS in relation to vitamin D status and metabolic disturbances is scarce. Six genetic linkage studies examined VDR and related polymorphisms in relation to PCOS $(41,42,43,44,45,46)$. The number of participants ranged from 56 to 690 . None of the 


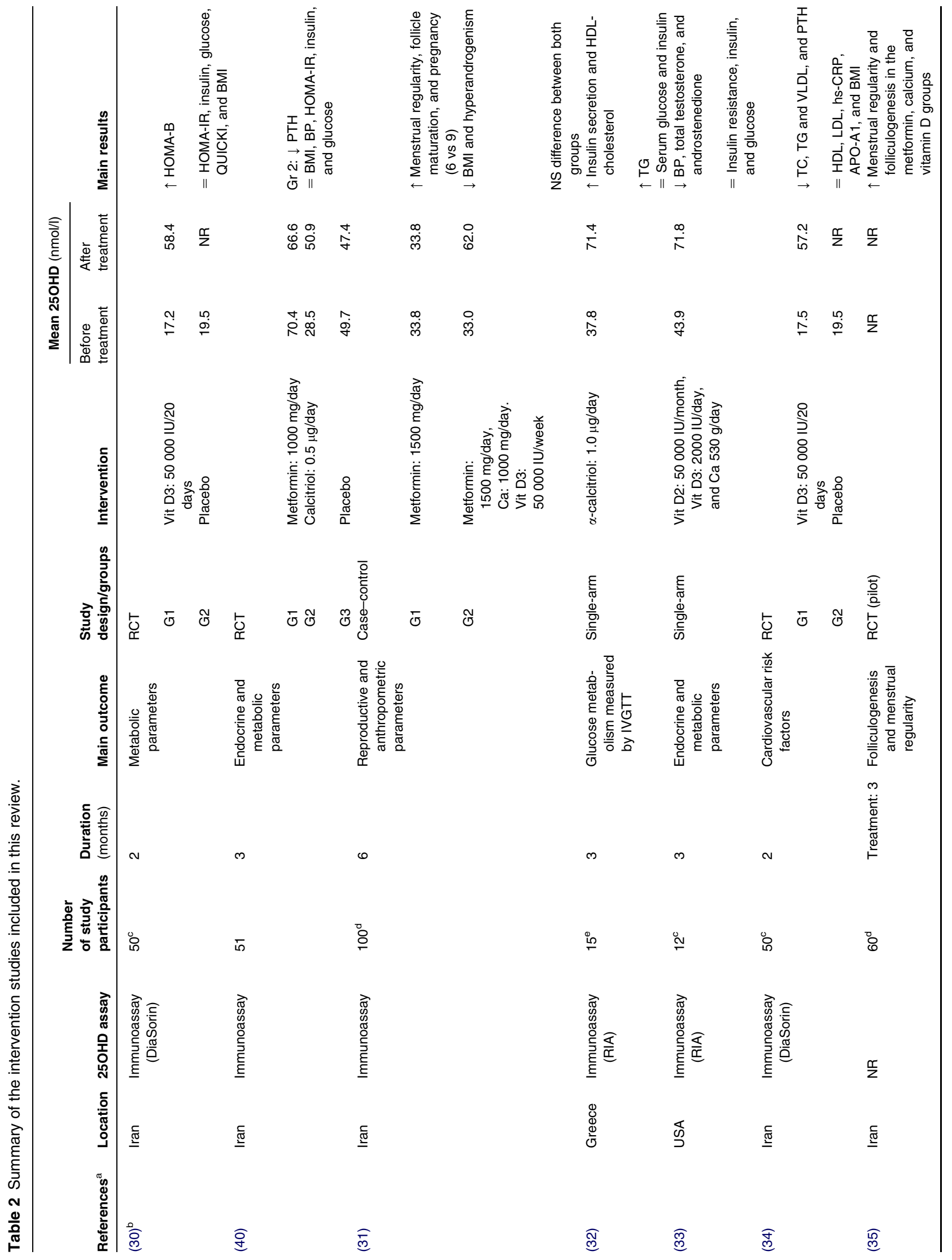




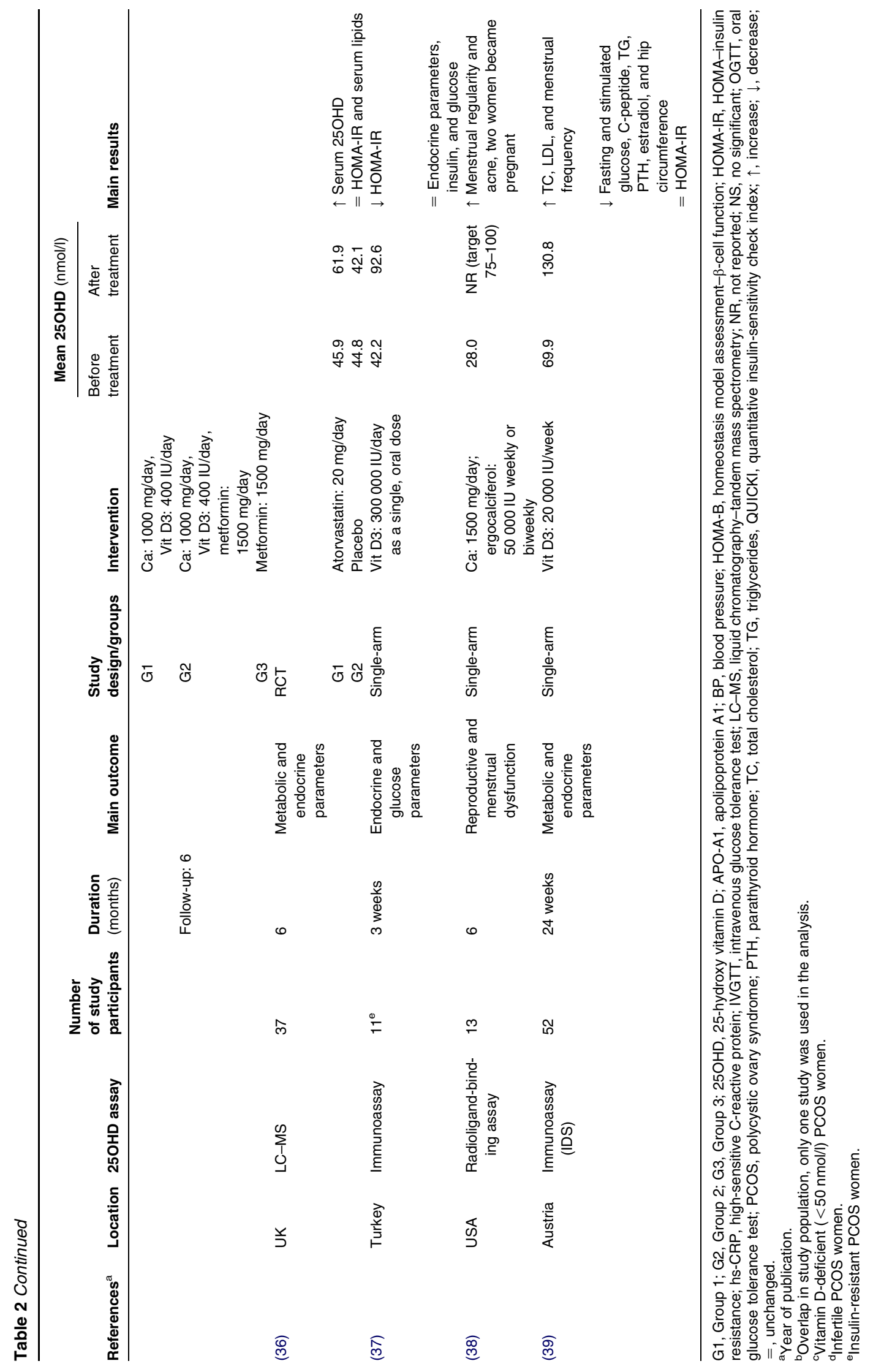

www.eje-online.org 
studies included a replication cohort. Besides singlenucleotide polymorphisms (SNPs) related to VDR genotype, SNPs in the insulin receptor (INSR), calcium-sensing receptor (CASR), parathyroid hormone $(P T H)$ receptor, adiponectin $(A D I P O Q)$ receptor, genes involved in cholesterol synthesis (DHCR7) and hydroxylation (CYP2R1), and genes involved in vitamin $\mathrm{D}$ transport (GC) were examined. A summary of the studies is given in Table 3.

Five studies determined the association between VDR polymorphisms and metabolic disturbances in women with $\operatorname{PCOS}(41,42,43,45,46)$.

In a cohort of 162 PCOS women and 162 control women, variants of VDR were found to be associated with an increase in insulin levels and IR: the VDR Apa-I genotype was found to be associated with the prevalence of PCOS (42). A study carried out by Wehr et al. (45) among 545 PCOS women and 145 control women found that the VDR $\mathrm{Cdx} 2$ 'AA' polymorphism was associated with lower fasting insulin levels $(P=0.039)$ and HOMA-IR $(P=0.041)$ compared with the other polymorphisms. These authors reported that the variants of both the GC gene, which encodes vitamin D-binding globulin, and DHRC7 gene, which encodes the enzyme 7-dehydrocholesterol reductase interacting with the synthetic pathway of vitamin D3, were associated with a significantly higher risk of vitamin D deficiency (OR 2.53 (1.27-5.06), $P=0.009$ and OR 2.66 (1.08-6.55), $P=0.033)$. Ranjzad et al. (43) found that the CASR 'TG' polymorphism was associated with IR.

Another study carried out in 181 Iranian PCOS women and 181 control women examined whether different polymorphisms in VDR, ADIPOQ, PTH, and ISNR genes were associated with PCOS. The authors found no difference in VDR gene polymorphisms between PCOS and control women (44). This result is in line with the results of the studies carried out by Lin et al. (41) and Zadeh-Vakili et al. (46).

\section{The correlation between vitamin D status, BMI, and IR in women with PCOS}

Vitamin $D$ status and IR In order to provide an overview of the relationship between vitamin D status and IR in women affected by PCOS compared with healthy control women, we carried out a regression analysis including serum $250 \mathrm{HD}$ as an independent

Table 3 Summary of the genetic linkage studies.

\begin{tabular}{|c|c|c|c|c|}
\hline References $^{a}$ & Location & $\begin{array}{l}\text { No. of study } \\
\text { participants }\end{array}$ & SNPs & Main results \\
\hline \multirow[t]{2}{*}{ (42) } & Iran & PCOS: 162 & $\begin{array}{l}\text { VDR (Bsm-I, Fok-I, Apa-I, and } \\
\text { Taq-I) polymorphisms }\end{array}$ & VDR Apa-I was associated with PCOS \\
\hline & & Control: 162 & & $\begin{array}{l}V D R \text { Fok-I 'FF' independently increased the } \\
\text { risk for insulin levels and insulin resistance in } \\
\text { PCOS }\end{array}$ \\
\hline$(45)$ & Austria & PCOS: 545 & $\begin{array}{l}\text { VDR (Cdx2, Bsm-I, Fok-I, Apa-I, } \\
\text { and Taq-I), GC } C^{\mathrm{a}}, D H C R 7^{\circ}, \text { and } \\
C Y P 2 R 1^{\mathrm{C}} \text { polymorphisms }\end{array}$ & $\begin{array}{l}\text { In PCOS women, VDR Cdx2 'AA' was } \\
\text { associated with lower fasting insulin levels } \\
\text { and HOMA-IR and higher QUICKI. GC 'GG' } \\
\text { and } D H C R 7 \text { ' } G G \text { ' were associated with a } \\
\text { higher risk of vitamin D deficiency }\end{array}$ \\
\hline (43) & Iran & PCOS: 181 & $\begin{array}{l}\text { INSR, } A D I P O Q, P T H, \text { and } V D R \\
\text { polymorphisms }\left(\text { SNPs }^{d}\right)\end{array}$ & $\begin{array}{l}\text { No significant difference was observed in } \\
\text { genotype and allele frequencies between } \\
\text { women with PCOS and control women }\end{array}$ \\
\hline \multirow[t]{2}{*}{ (44) } & Iran & $\begin{array}{l}\text { Control: } 145 \\
\text { PCOS: } 56\end{array}$ & $\begin{array}{l}\text { VDR (Bsm-I, Fok-I, Apa-I, Taq-I, } \\
\text { and Tru9-I), PTH, CASR, INSR, } \\
\text { and ADIPOQ polymorphisms }\end{array}$ & $\begin{array}{l}\text { CASR 'TG' was associated with higher } \\
\text { HOMA-IR }\end{array}$ \\
\hline & & Control: 181 & & $\begin{array}{l}\text { VDR Taq-I was associated with elevated } \\
\text { serum LH levels } \\
\text { VDR Bsm-I and ADIPOQ (rs1501299) were } \\
\text { associated with decreased SHBG levels }\end{array}$ \\
\hline \multirow[t]{2}{*}{$(46)$} & Iran & PCOS: 260 & VDR (Tru9-I) & $\begin{array}{l}\text { No significant difference was observed in } \\
\text { genotype and allele frequencies between } \\
\text { women with PCOS and control women }\end{array}$ \\
\hline & & Control: 221 & & \\
\hline
\end{tabular}

ADIPOQ, adiponectin; CaSR, calcium-sensing receptor; HOMA-IR, homeostasis model assessment-insulin resistance; INSR, insulin receptor; NR, not reported; PCOS, polycystic ovary syndrome; PTH, parathyroid hormone; QUICKI, quantitative insulin-sensitivity check index; SHBG, sex hormone-binding globulin; SNPs, single-nucleotide polymorphisms; VDR, vitamin D receptor.

ancodes vitamin D-binding globulin.

${ }^{b}$ Encodes the enzyme 7-dehydrocholesterol reductase, interacting with the synthetic pathway of vitamin D3.

${ }^{\mathrm{c}}$ Encodes a hepatic microsomal enzyme, hypothetically involved in the 25-hydroxylation of vitamin $\mathrm{D}$ in the liver.

dINSR gene (rs2059806 and rs1799817), ADIPOQ gene (rs2241766 and rs1501299), PTH gene (rs6256), and VDR gene (rs757343).

${ }^{\mathrm{e}} \mathrm{PTH}$ (rs6256), CASR (rs1801725), INSR (rs2059806 and rs1799817), and ADIPOQ (rs2241766 and rs1501299). 


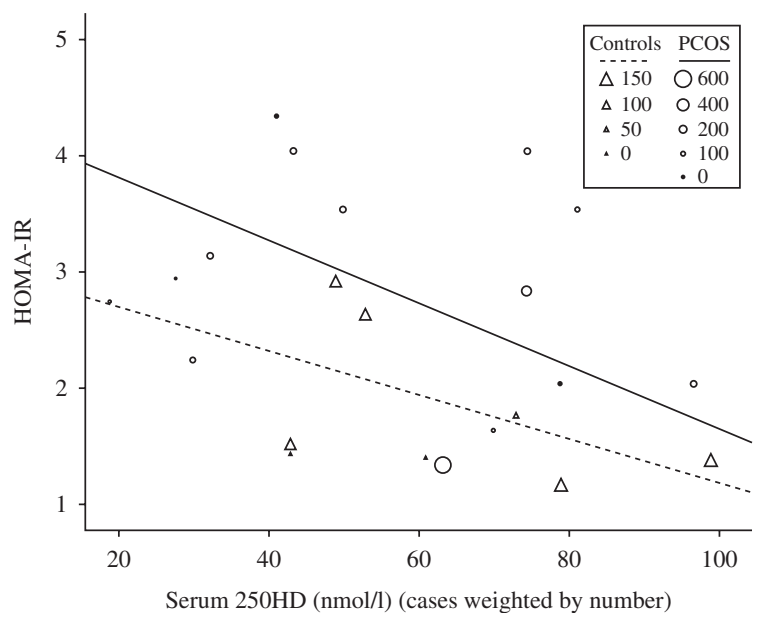

Figure 2 Association between vitamin $D$ status and insulin resistance in PCOS women $(18,19,20,22,23,24,26,27,28,29$, $30,33,37,39,40,41,43,45)$ and control women $(19,20,22,23,24$ $27,28,41,45)$. The circles and unbroken line represent PCOS women and the triangles and broken line represent control women. In the univariate regression analysis, the slope of the line was -0.03 for PCOS women and -0.02 for control women. The size of the symbols indicates the number of participants included in the corresponding study.

variable and HOMA-IR as a dependent variable. Of the 29 reviewed studies, 18 examined this correlation and reported the mean and/or median values and were therefore included in this analysis $(18,19,20,22,23$, $24,26,27,28,29,30,33,37,39,40,41,43,45)$. Nine studies reported these values also for control women $(19,20,22,23,24,27,28,41,45)$. The data were weighted by the number of participants in each study, resulting in a total of 1893 PCOS women and 717 control women. The overall mean of serum $25 \mathrm{OHD}$ was $61.2 \mathrm{nmol} / \mathrm{l}$ in PCOS women and $67.1 \mathrm{nmol} / \mathrm{l}$ in control women. The overall mean of HOMA-IR was 2.71 in PCOS women and 1.8 in control women. Univariate regression analysis revealed that an increase in serum 250HD levels was significantly associated with a decrease in HOMA-IR in both PCOS women $\left(B_{1}=-0.027 ; \mathrm{CI}-0.030\right.$ to $\left.-0.024 ; P \leq 0.001\right)$ and control women $\left(B_{1}=-0.019\right.$; $\mathrm{CI}-0.021$ to -0.017 ; $P \leq 0.001$ ) (Fig. 2). The results indicate that every $10 \mathrm{nmol} / \mathrm{l}$ increase in serum 250HD levels decreases HOMA-IR by 0.27 in PCOS women and 0.19 in control women. After multivariate regression analysis with serum 25OHD and BMI as independent variables, serum vitamin $\mathrm{D}$ was found to no longer be an independent predictor of IR in PCOS women. The result remained significant for control women.

Vitamin D status and BMI Data on the association between anthropometric parameters and serum $250 \mathrm{HD}$ were provided in 23 of the 29 reviewed studies $(18,19$, $20,21,22,23,24,26,27,28,29,30,31,32,33,34$, $36,37,38,39,41,43,45)$, and nine studies reported these data also for control women $(19,20,22,23,24$, $27,28,41,45)$. After regression analyses of the weighted means including a total of 2045 PCOS women and 717 control women, BMI appeared to be an independent predictor of serum 250HD in both PCOS and control women $\left(B_{1}=-4.5, P<0.001\right.$ and $B_{1}=-8.4, P<0.001$; Fig. 3). The overall mean of BMI in these studies was $26.6 \mathrm{~kg} / \mathrm{m}^{2}$ in PCOS women and $25.1 \mathrm{~kg} / \mathrm{m}^{2}$ in control women.

BMI and IR Nineteen of the included studies investigated the correlation between BMI and HOMA-IR in PCOS women $(18,19,20,22,23,24,25,26,27,28$, $29,30,33,37,39,41,43,45,46)$ and nine in control women $(19,20,22,23,24,27,28,41,45)$. All the studies revealed a significant association between BMI and HOMA-IR. A total of 2311 PCOS women and 717 control women participated in these studies. Multivariate regression analyses with serum 250HD and BMI as independent variables and HOMA-IR as a dependent variable revealed that BMI was an independent predictor of HOMA-IR in PCOS and control women $\left(B_{1}=0.41, P<0.001\right.$ and $B_{1}=0.10, P<0.001$ respectively; Fig. 4).

\section{Discussion}

This systematic review was carried out to examine the role of vitamin $\mathrm{D}$ status and polymorphisms in metabolic disturbances, in particular IR, in women suffering from PCOS. There was inconsistency in the

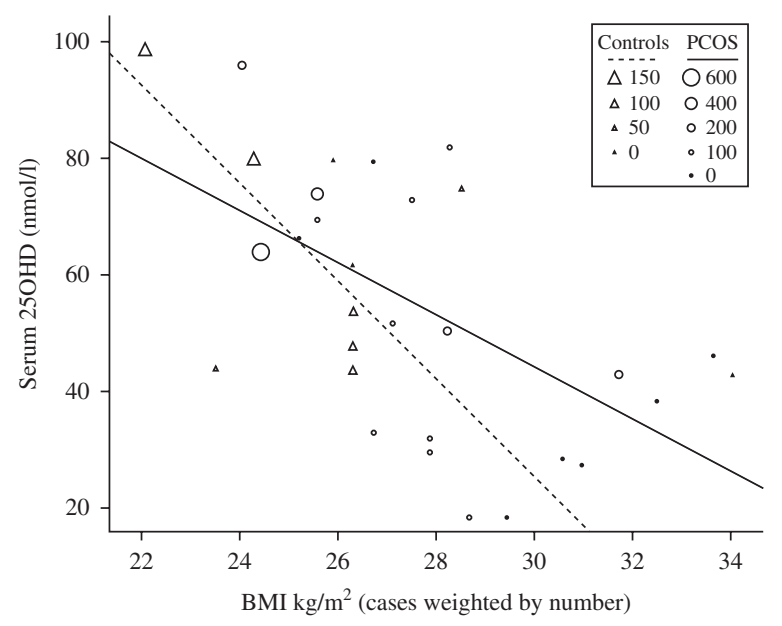

Figure 3 Association between BMI and vitamin D status in PCOS women $(18,19,20,21,22,23,24,26,27,28,29,30,31,32,33$, $34,36,37,38,39,41,43,45)$ and control women $(19,20,22,23$, $24,27,28,41,45)$. The circles and unbroken line represent PCOS women and the triangles and broken line represent control women. In the univariate regression analysis, the slope of the line was -4.5 for PCOS women and -8.4 for control women. The size of the symbols indicates the number of participants included in the corresponding study. 


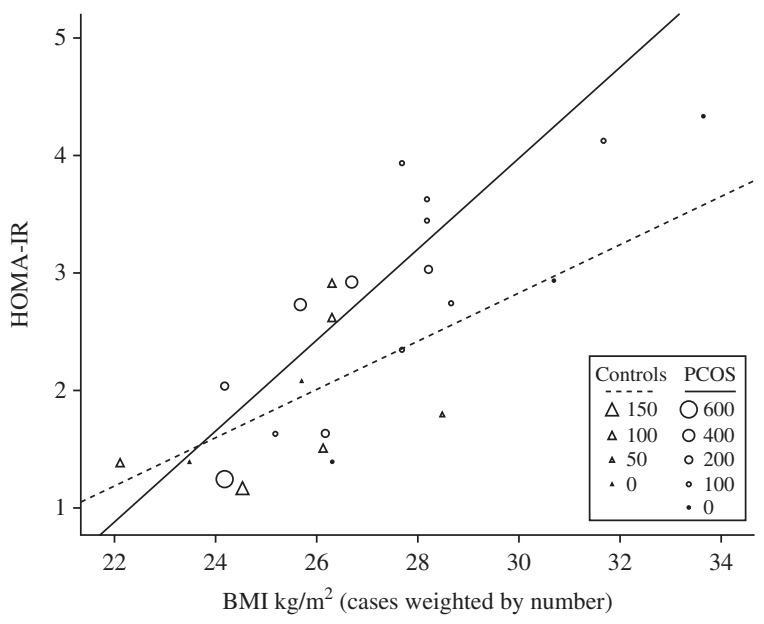

Figure 4 Association between $\mathrm{BMI}$ and insulin resistance in PCOS women $(18,19,20,22,23,24,25,26,27,29,30,33,37,39,41,42$, $43,45,46)$ and control women $(19,20,22,23,24,27,28,41,45)$. The circles and unbroken line represent PCOS women and the triangles and broken line represent control women. In the univariate regression analysis, the slope of the line was 0.41 for PCOS women and 0.10 for control women. The size of the symbols indicates the number of participants included in the corresponding study.

results of the studies analyzing the relationship between vitamin D status and metabolic disturbances in PCOS women. These conflicting findings might be due to the small sample sizes, the lack of adjustments for confounders, the use of different definitions for PCOS, the use of different assays for serum 250HD measurement, the duration of intervention, the use of different amounts of vitamin D supplementation in the intervention trials, and the lack of an optimal serum 250HD level in the general population. Only one well-designed randomized placebo-controlled trial demonstrating no effect of vitamin D3 supplementation on IR has been carried out until now (30). The small sample size and the relatively short follow-up duration could account for the lack of an effect. Another RCT carried out among 51 PCOS women by Bonakdaran et al. (40) did report a decrease in HOMA-IR after 3 months of calcitriol supplementation. However, this decrease was not significant probably due to the small sample size.

The relationship between obesity, vitamin D status, and IR has not been clarified yet. There is an ongoing discussion on whether vitamin D deficiency and IR are causally interrelated or whether they are both BMI dependent. In this review, we provided an overview of the correlation between these variables by carrying out a linear regression analysis. The univariate linear regression analysis revealed both serum vitamin D and BMI to be independent predictors of IR. However, in the multivariate analysis, serum 250HD was no longer an independent predictor of IR in PCOS women. A strong independent relationship was observed between BMI as an explanatory variable and serum $25 \mathrm{OHD}$ as an outcome variable. A limitation of the data analyses conducted in this review is that the weighted means of the reviewed studies instead of the original data were used. Another important point to be considered is the different methodologies used for vitamin D measurement in the reviewed studies. At present, many discussions are going on as to which test is the most reliable one for vitamin D measurement. Owing to the increasing recognition of the high prevalence and diverse consequences of vitamin D deficiency, a massive increase in vitamin $\mathrm{D}$ testing is being observed worldwide. A recent trial has demonstrated the limitation of different assays (47). Both could have influenced the results.

The demonstrated inverse relationship between BMI and vitamin D status has been established in earlier reports $(48,49)$. It has been shown that in obese individuals a higher proportion of vitamin $\mathrm{D}$, which is fat soluble, is sequestered in adipose tissue and thereby the bioavailability of vitamin D is low. Alternatively, obese individuals tend to spend less time outdoors exposed to sunlight, leading to insufficient biosynthesis of vitamin D generated through the skin. Moreover, obesity is highly prevalent in women affected by PCOS, with the highest prevalence being reported in studies conducted in the USA and Australia, with $61-76 \%$ of women with PCOS being considered obese and $85 \%$ considered overweight or obese (1). In addition, obesity has a strong correlation with IR and type 2 diabetes mellitus (50). This association is in line with our results. Despite the strong association between obesity and IR, multiple studies have demonstrated that women affected by PCOS have more severe IR than expected on the basis of their body weight $(51,52,53)$. In this regard, vitamin $\mathrm{D}$ status has more than once been designated as a possible contributing factor for IR in women affected by PCOS. This hypothesis is supported by animal studies with $V d r$ null mice and by human models (10). The largest cross-sectional study (NHANES data) has demonstrated an inverse correlation between serum 250HD concentrations and diabetes prevalence. After multivariate adjustment, serum 250HD concentrations were found to be negatively correlated with IR (15). Pittas et al. (54) confirmed these results in a 3-year follow-up study in which participants with impaired fasting glucose levels who used cholecalciferol plus calcium supplementation had a smaller increase in IR compared with controls who received a placebo.

The potential mechanisms by which vitamin D can affect glucose metabolism could be the result of direct and indirect actions of serum 250HD: i) direct stimulation of insulin release through the expression of VDR as well as the enzyme $1 \alpha$-OHase in the pancreatic $\beta$-cells; ii) through the binding of the $1,25(\mathrm{OH}) 2 \mathrm{D}-\mathrm{VDR}$ complex to the vitamin D response element of the INSR at the tissue level and thereby enhancing insulin responsiveness for glucose transport; and iii) suppression of the release of proinflammatory cytokines that are believed to mediate IR (11). The latter 
mechanism is supported by studies showing an association between low serum 250HD levels and increased C-reactive protein levels $(25,55)$. Vitamin D may play an indirect role through its influence on the extracellular and intracellular calcium regulation, which is essential for the mediation of glucose transport in target tissues.

Nine of the reviewed studies compared PCOS women with control women. An important observation is that vitamin $\mathrm{D}$ status did not markedly differ between both the groups (overall mean $61.2 \mathrm{nmol} / \mathrm{l}$ in PCOS women vs $67.1 \mathrm{nmol} / \mathrm{l}$ in control women). It is remarkable that despite a slight difference in mean BMI between both the groups, HOMA-IR differed considerably, suggesting that PCOS per se is a risk factor for the development of IR. This is in line with some but not all earlier results $(8,9,53)$. By contrast, PCOS per se does not seem to be a risk factor for vitamin D deficiency. The studies carried out by Ngo et al., Panidis et al., and Mahmoudi et al. even found a higher serum $250 \mathrm{HD}$ level in PCOS women than in control women $(20,22,23)$. However, these data were not adjusted for the season of measurement and diet.

Another important note is that the inconsistent results in the reviewed articles may be explained by the hypothesis that vitamin D supplementation may only be effective on IR in case a significant IR exists. As the majority of the reviewed studies did not include diabetic PCOS women, this could be a possible explanation why an effect of vitamin D supplementation on IR was not evident in all studies. However, the RCT carried out by Ardabili et al. did not report an effect of vitamin D supplementation, despite HOMA-IR of 3.17 in PCOS women (30).

Another hypothesis for the lack of an effect may be that baseline serum 250HD is already sufficient. It seems plausible that in case of an adequate vitamin D status no effect can be observed after vitamin D supplementation. In this regard, it should be noted that the optimal serum $250 H D$ level is not clear until now. Although there is no consensus on optimal serum $250 \mathrm{HD}$ levels, frequently vitamin D deficiency is defined by a serum $250 \mathrm{HD}$ level $<25 \mathrm{nmol} / \mathrm{l}(10 \mathrm{ng} / \mathrm{ml})$. Serum $250 H D$ levels between 25 and $50 \mathrm{nmol} / \mathrm{l}(10-20 \mathrm{ng} / \mathrm{ml})$ are considered to indicate vitamin D insufficiency, and serum 250HD levels $>50 \mathrm{nmol} / \mathrm{l}$ are considered to indicate vitamin sufficiency. Recently, the Institute of Medicine, reviewing all the evidence, has set the required serum $250 \mathrm{HD}$ levels at $50 \mathrm{nmol} / \mathrm{l}$ (56), but expert opinions remain divided. In addition, no optimal treatment regimen has been established yet for the treatment of vitamin D deficiency in different populations. In this regard, this may influence the study results, as all the reviewed intervention studies used different amounts and durations of vitamin D supplementation.

In conclusion, the relationship between vitamin $\mathrm{D}$ status and metabolic disturbances in women with PCOS remains unclear. Current literature suggests an inverse correlation between vitamin D status and IR in women with PCOS. However, due to the heterogeneity of the studies, it is hard to draw an informed conclusion. Future research with adequately powered randomized placebo-controlled double-blind studies of vitamin D supplementation in women affected by PCOS is warranted. Until then, screening women who are at risk of vitamin D deficiency and supplementation with vitamin $\mathrm{D}$ could be considered.

\section{Supplementary data}

This is linked to the online version of the paper at http://dx.doi.org/ 10.1530/EJE-13-0617.

\section{Declaration of interest}

J S E Laven has received fees and grant support from the following companies (in alphabetic order): Ferring, Genovum, Merck-Serono, Organon, Schering Plough, and Serono. The other authors have no conflicts of interest to declare.

\section{Funding}

This research did not receive any specific grant from any funding agency in the public, commercial or not-for-profit sector.

\section{Author contribution statement}

Each author contributed in a substantial way to the work described in the manuscript and its preparation. Y H M Krul-Poel and C Snackey were responsible for the search and selection of articles, extraction of data, and writing and revision of the manuscript. S Simsek initiated the study, contributed to the study design, and revised the paper critically for important content. Y Louwers, J S E Laven, P Lips, and C B Lambalk revised the paper critically. Y H M Krul-Poel and Y Louwers were responsible for the statistical analyses. C Snackey was responsible for the figures. All the authors were involved in the final approval of the version to be published.

\section{References}

1 Fauser BC, Tarlatzis BC, Rebar RW, Legro RS, Balen AH, Lobo R, Carmina E, Chang J, Yildiz BO, Laven JS et al. Consensus on women's health aspects of polycystic ovary syndrome (PCOS): the Amsterdam ESHRE/ASRM-Sponsored 3rd PCOS Consensus Workshop Group. Fertility and Sterility 201297 28-38. (doi:10.1016/j. fertnstert.2011.09.024)

2 de Groot PC, Dekkers OM, Romijn JA, Dieben SW \& Helmerhorst FM. PCOS, coronary heart disease, stroke and the influence of obesity: a systematic review and meta-analysis. Human Reproduction Update 201117 495-500. (doi:10.1093/ humupd/dmr001)

3 Diamanti-Kandarakis E \& Dunaif A. Insulin resistance and the polycystic ovary syndrome revisited: an update on mechanisms and implications. Endocrine Reviews 201233 981-1030. (doi:10.1210/er.2011-1034)

4 Wild RA, Rizzo M, Clifton S \& Carmina E. Lipid levels in polycystic ovary syndrome: systematic review and meta-analysis. Fertility and Sterility 201195 1073-1079. (doi:10.1016/j.fertnstert. 2010.12.027)

5 Ehrmann DA, Barnes RB, Rosenfield RL, Cavaghan MK \& Imperial J. Prevalence of impaired glucose tolerance and diabetes in women with polycystic ovary syndrome. Diabetes Care 199922 141-146. (doi:10.2337/diacare.22.1.141) 
6 Moran LJ, Misso ML, Wild RA \& Norman RJ. Impaired glucose tolerance, type 2 diabetes and metabolic syndrome in polycystic ovary syndrome: a systematic review and meta-analysis. Human Reproduction Update 201016 347-363. (doi:10.1093/humupd/ dmq001)

7 Norman RJ, Dewailly D, Legro RS \& Hickey TE. Polycystic ovary syndrome. Lancet $2007 \mathbf{3 7 0}$ 685-697. (doi:10.1016/S01406736(07)61345-2)

8 Dunaif A, Segal KR, Futterweit W \& Dobrjansky A. Profound peripheral insulin resistance, independent of obesity, in polycystic ovary syndrome. Diabetes 198938 1165-1174. (doi:10.2337/ diab.38.9.1165)

9 Ketel IJ, Serne EH, Ijzerman RG, Korsen TJ, Twisk JW, Hompes PG, Smulders YM, Homburg R, Vorstermans L, Stehouwer CD et al. Insulin-induced capillary recruitment is impaired in both lean and obese women with PCOS. Human Reproduction 201126 31303137. (doi:10.1093/humrep/der296)

10 Bouillon R, Carmeliet G, Verlinden L, van Etten E, Verstuyf A, Luderer HF, Lieben L, Mathieu C \& Demay M. Vitamin D and human health: lessons from vitamin D receptor null mice. Endocrine Reviews 200829 726-776. (doi:10.1210/er.2008-0004)

11 Alvarez JA \& Ashraf A. Role of vitamin D in insulin secretion and insulin sensitivity for glucose homeostasis. International Journal of Endocrinology 20102010351385 . (doi:10.1155/2010/351385)

12 Chiu KC, Chu A, Go VL \& Saad MF. Hypovitaminosis D is associated with insulin resistance and $\beta$ cell dysfunction. American Journal of Clinical Nutrition 200479 820-825.

13 Ford ES, Ajani UA, McGuire LC \& Liu S. Concentrations of serum vitamin D and the metabolic syndrome among U.S. adults. Diabetes Care 200528 1228-1230. (doi:10.2337/diacare.28.5.1228)

14 Pittas AG, Lau J, Hu FB \& Dawson-Hughes B. The role of vitamin D and calcium in type 2 diabetes. A systematic review and metaanalysis. Journal of Clinical Endocrinology and Metabolism 200792 2017-2029. (doi:10.1210/jc.2007-0298)

15 Scragg R, Sowers M \& Bell C. Serum 25-hydroxyvitamin D, diabetes, and ethnicity in the Third National Health and Nutrition Examination Survey. Diabetes Care $2004 \quad 27$ 2813-2818. (doi:10.2337/diacare.27.12.2813)

16 Thomson RL, Spedding S \& Buckley JD. Vitamin D in the aetiology and management of polycystic ovary syndrome. Clinical Endocrinology 201277 343-350. (doi:10.1111/j.1365-2265.2012. 04434.x)

17 Rotterdam ESHRE/ASRM-Sponsored PCOS consensus workshop group. Revised 2003 consensus on diagnostic criteria and longterm health risks related to polycystic ovary syndrome (PCOS). Human Reproduction 200419 41-47. (doi:10.1093/humrep/ deh098)

18 Hahn S, Haselhorst U, Tan S, Quadbeck B, Schmidt M, Roesler S, Kimmig R, Mann K \& Janssen OE. Low serum 25-hydroxyvitamin D concentrations are associated with insulin resistance and obesity in women with polycystic ovary syndrome. Experimental and Clinical Endocrinology $\mathcal{E}$ Diabetes 2006114 577-583. (doi:10.1055/s-2006948308)

19 Li HW, Brereton RE, Anderson RA, Wallace AM \& Ho CK. Vitamin D deficiency is common and associated with metabolic risk factors in patients with polycystic ovary syndrome. Metabolism 201160 1475-1481. (doi:10.1016/j.metabol.2011.03.002)

20 Mahmoudi T, Gourabi H, Ashrafi M, Yazdi RS \& Ezabadi Z. Calciotropic hormones, insulin resistance, and the polycystic ovary syndrome. Fertility and Sterility 201093 1208-1214. (doi:10.1016/j.fertnstert.2008.11.031)

21 Muscogiuri G, Policola C, Prioletta A, Sorice G, Mezza T, Lassandro A, Della CS, Pontecorvi A \& Giaccari A. Low levels of 25(OH)D and insulin-resistance: 2 unrelated features or a causeeffect in PCOS? Clinical Nutrition 201231 476-480. (doi:10.1016/j.clnu.2011.12.010)

22 Ngo DT, Chan WP, Rajendran S, Heresztyn T, Amarasekera A, Sverdlov AL, O'Loughlin PD, Morris HA, Chirkov YY, Norman RJ et al. Determinants of insulin responsiveness in young women: impact of polycystic ovarian syndrome, nitric oxide, and vitamin D. Nitric Oxide 201125 326-330. (doi:10.1016/j.niox.2011.06.005)
23 Panidis D, Balaris C, Farmakiotis D, Rousso D, Kourtis A, Balaris V, Katsikis I, Zournatzi V \& Diamanti-Kandarakis E. Serum parathyroid hormone concentrations are increased in women with polycystic ovary syndrome. Clinical Chemistry $2005 \mathbf{5 1}$ 1691-1697. (doi:10.1373/clinchem.2005.052761)

24 Savastano S, Valentino R, Di Somma C, Orio F, Pivonello C, Passaretti F, Brancato V, Formisano P, Colao A, Beguinot F et al. Serum 25-hydroxyvitamin D Levels, phosphoprotein enriched in diabetes gene product (PED/PEA-15) and leptin-to-adiponectin ratio in women with PCOS. Nutrition and Metabolism 2011884. (doi:10.1186/1743-7075-8-84)

25 Wehr E, Pilz S, Schweighofer N, Giuliani A, Kopera D, Pieber TR \& Obermayer-Pietsch B. Association of hypovitaminosis D with metabolic disturbances in polycystic ovary syndrome. European Journal of Endocrinology 2009161 575-582. (doi:10.1530/EJE09-0432)

26 Yildizhan R, Kurdoglu M, Adali E, Kolusari A, Yildizhan B, Sahin HG \& Kamaci M. Serum 25-hydroxyvitamin D concentrations in obese and non-obese women with polycystic ovary syndrome. Archives of Gynecology and Obstetrics $2009 \mathbf{2 8 0}$ 559-563. (doi:10.1007/s00404-009-0958-7)

27 Hassan NE, El Orabi HA, Eid YM \& Mohammed NR. Effect of 25-hydroxyvitamin D on metabolic parameters and insulin resistance in patients with polycystic ovarian syndrome. Middle East Fertility Society Journal 201217 176-180. (doi:10.1016/j. mefs.2012.04.005)

28 Mazloomi S, Sharifi F, Hajihosseini R, Kalantari S \& Mazloomzadeh S. Association between hypoadiponectinemia and low serum concentrations of calcium and vitamin D in women with polycystic ovary syndrome. ISRN Endocrinology 20122012 949427. (doi:10.5402/2012/949427)

29 Patra SK, Nasrat H, Goswami B \& Jain A. Vitamin D as a predictor of insulin resistance in polycystic ovarian syndrome. Diabetes $\mathcal{E}$ Metabolic Syndrome 20126 146-149. (doi:10.1016/j.dsx.2012. 09.006)

30 Ardabili HR, Gargari BP \& Farzadi L. Vitamin D supplementation has no effect on insulin resistance assessment in women with polycystic ovary syndrome and vitamin D deficiency. Nutrition Research 201232 195-201. (doi:10.1016/j.nutres.2012.02. 001)

31 Firouzabadi R, Aflatoonian A, Modarresi S, Sekhavat L \& Mohammad Taheri S. Therapeutic effects of calcium \& vitamin D supplementation in women with PCOS. Complementary Therapies in Clinical Practice 201218 85-88. (doi:10.1016/j.ctcp.2012.01.005)

32 Kotsa K, Yavropoulou MP, Anastasiou O \& Yovos JG. Role of vitamin D treatment in glucose metabolism in polycystic ovary syndrome. Fertility and Sterility 200992 1053-1058. (doi:10.1016/j.fertnstert.2008.07.1757)

33 Pal L, Berry A, Coraluzzi L, Kustan E, Danton C, Shaw J \& Taylor H. Therapeutic implications of vitamin D and calcium in overweight women with polycystic ovary syndrome. Gynecological Endocrinology $2012 \mathbf{2 8}$ 965-968. (doi:10.3109/09513590.2012. 696753)

34 Rahimi-Ardabili H, Pourghassem GB \& Farzadi L. Effects of vitamin D on cardiovascular disease risk factors in polycystic ovary syndrome women with vitamin D deficiency. Journal of Endocrinological Investigation 201336 28-32. (doi:10.3275/ 8303)

35 Rashidi B, Haghollahi F, Shariat M \& Zayerii F. The effects of calcium-vitamin D and metformin on polycystic ovary syndrome: a pilot study. Taiwanese Journal of Obstetrics $\mathcal{E}$ Gynecology 200948 142-147. (doi:10.1016/S1028-4559(09)60275-8)

36 Sathyapalan T, Shepherd J, Arnett C, Coady AM, Kilpatrick ES \& Atkin SL. Atorvastatin increases 25-hydroxy vitamin D concentrations in patients with polycystic ovary syndrome. Clinical Chemistry 201056 1696-1700. (doi:10.1373/clinchem.2010. 144014)

37 Selimoglu H, Duran C, Kiyici S, Ersoy C, Guclu M, Ozkaya G, Tuncel E, Erturk E \& Imamoglu S. The effect of vitamin D 
replacement therapy on insulin resistance and androgen levels in women with polycystic ovary syndrome. Journal of Endocrinological Investigation 201033 234-238. (doi:10.3275/6560)

38 Thys-Jacobs S, Donovan D, Papadopoulos A, Sarrel P \& Bilezikian JP. Vitamin D and calcium dysregulation in the polycystic ovarian syndrome. Steroids $1999 \quad \mathbf{6 4} \quad 430-435$. (doi:10.1016/S0039-128X(99)00012-4)

39 Wehr E, Pieber TR \& Obermayer-Pietsch B. Effect of vitamin D3 treatment on glucose metabolism and menstrual frequency in polycystic ovary syndrome women: a pilot study. Journal of Endocrinological Investigation 201134 757-763. (doi:10.3275/7748)

40 Bonakdaran S, Khorasani ZM, Davachi B \& Khorasani JM. The effects of calcitriol on improvement of insulin resistance, ovulation and comparison with metformin therapy in PCOS patients: a randomized placebo-controlled clinical trial. Iranian Journal of Reproductive Medicine 201210 465-472.

41 Lin MW, Tsai SJ, Chou PY, Huang MF, Sun HS \& Wu MH. Vitamin D receptor 1a promoter $-1521 \mathrm{G} / \mathrm{C}$ and $-1012 \mathrm{~A} / \mathrm{G}$ polymorphisms in polycystic ovary syndrome. Taiwanese Journal of Obstetrics \& Gynecology 201251 565-571. (doi:10.1016/j.tjog. 2012.09.011)

42 Mahmoudi T. Genetic variation in the vitamin D receptor and polycystic ovary syndrome risk. Fertility and Sterility 200992 1381-1383. (doi:10.1016/j.fertnstert.2009.05.002)

43 Ranjzad F, Mahban A, Shemirani AI, Mahmoudi T, Vahedi M, Nikzamir A \& Zali MR. Influence of gene variants related to calcium homeostasis on biochemical parameters of women with polycystic ovary syndrome. Journal of Assisted Reproduction and Genetics 201128 225-232. (doi:10.1007/s10815-010-9506-4)

44 Ranjzad F, Mahmoudi T, Irani SA, Mahban A, Nikzamir A, Vahedi M, Ashrafi M \& Gourabi H. A common variant in the adiponectin gene and polycystic ovary syndrome risk. Molecular Biology Reports 201239 2313-2319. (doi:10.1007/s11033011-0981-1)

45 Wehr E, Trummer O, Giuliani A, Gruber HJ, Pieber TR \& Obermayer-Pietsch B. Vitamin D-associated polymorphisms are related to insulin resistance and vitamin D deficiency in polycystic ovary syndrome. European Journal of Endocrinology $2011 \mathbf{1 6 4}$ 741-749. (doi:10.1530/EJE-11-0134)

46 Zadeh-Vakili A, Ramezani TF, Daneshpour MS, Zarkesh M, Saadat N \& Azizi F. Genetic polymorphism of vitamin D receptor gene affects the phenotype of PCOS. Gene 2013515 193-196. (doi:10.1016/j.gene.2012.11.049)

47 Farrell CJ, Martin S, McWhinney B, Straub I, Williams P \& Herrmann M. State-of-the-art vitamin D assays: a comparison of automated immunoassays with liquid chromatography-tandem mass spectrometry methods. Clinical Chemistry $2012 \mathbf{5 8}$ 531-542. (doi:10.1373/clinchem.2011.172155)
48 Snijder MB, van Dam RM, Visser M, Deeg DJ, Dekker JM, Bouter LM, Seidell JC \& Lips P. Adiposity in relation to vitamin D status and parathyroid hormone levels: a population-based study in older men and women. Journal of Clinical Endocrinology and Metabolism 200590 4119-4123. (doi:10.1210/jc.2005-0216)

49 Tzotzas T, Papadopoulou FG, Tziomalos K, Karras S, Gastaris K, Perros P \& Krassas GE. Rising serum 25-hydroxy-vitamin D levels after weight loss in obese women correlate with improvement in insulin resistance. Journal of Clinical Endocrinology and Metabolism 201095 4251-4257. (doi:10.1210/jc.2010-0757)

50 Salans LB, Knittle JL \& Hirsch J. The role of adipose cell size and adipose tissue insulin sensitivity in the carbohydrate intolerance of human obesity. Journal of Clinical Investigation 1968 47 153-165. (doi:10.1172/JCI105705)

51 Dunaif A \& Finegood DT. $\beta$-Cell dysfunction independent of obesity and glucose intolerance in the polycystic ovary syndrome. Journal of Clinical Endocrinology and Metabolism $1996 \mathbf{8 1}$ 942-947. (doi:10.1210/jc.81.3.942)

52 Dunaif A. Insulin resistance and the polycystic ovary syndrome: mechanism and implications for pathogenesis. Endocrine Reviews 199718 774-800. (doi:10.1210/er.18.6.774)

53 Ketel IJ, Stehouwer CD, Serne EH, Korsen TJ, Hompes PG, Smulders YM, de Jongh RT, Homburg R \& Lambalk CB. Obese but not normal-weight women with polycystic ovary syndrome are characterized by metabolic and microvascular insulin resistance. Journal of Clinical Endocrinology and Metabolism 2008 93 3365-3372. (doi:10.1210/jc.2008-0626)

54 Pittas AG, Harris SS, Stark PC \& Dawson-Hughes B. The effects of calcium and vitamin D supplementation on blood glucose and markers of inflammation in nondiabetic adults. Diabetes Care 2007 30 980-986. (doi:10.2337/dc06-1994)

55 Amer M \& Qayyum R. Relation between serum 25-hydroxyvitamin D and C-reactive protein in asymptomatic adults (from the continuous National Health and Nutrition Examination Survey 2001 to 2006). American Journal of Cardiology 2012109 226-230. (doi:10.1016/j.amjcard.2011.08.032)

56 Ross AC, Manson JE, Abrams SA, Aloia JF, Brannon PM, Clinton SK, Durazo-Arvizu RA, Gallagher JC, Gallo RL, Jones G et al. The 2011 report on dietary reference intakes for calcium and vitamin D from the Institute of Medicine: what clinicians need to know. Journal of Clinical Endocrinology and Metabolism 201196 53-58. (doi:10.1210/jc.2010-2704)

Received 29 July 2013

Revised version received 10 September 2013

Accepted 17 September 2013 